\title{
Heralded Single-Magnon Quantum Memory for Photon Polarization States
}

\author{
Haruka Tanji, ${ }^{1,2}$ Saikat Ghosh, ${ }^{2}$ Jonathan Simon, ${ }^{1,2}$ Benjamin Bloom, ${ }^{2}$ and Vladan Vuletić ${ }^{2}$ \\ ${ }^{1}$ Department of Physics, Harvard University, Cambridge, Massachusetts 02138, USA \\ ${ }^{2}$ Department of Physics, MIT-Harvard Center for Ultracold Atoms, and Research Laboratory of Electronics, \\ Massachusetts Institute of Technology, Cambridge, Massachusetts 02139, USA
}

(Received 26 August 2008; published 20 July 2009)

\begin{abstract}
We demonstrate a heralded quantum memory where a photon announces the mapping of a light polarization state onto a single collective-spin excitation (magnon) shared between two atomic ensembles. The magnon can be converted at a later time into a single polarized photon with polarization fidelity over 90(2)\% for all fiducial input states, well above the classical limit of $\frac{2}{3}$. The process can be viewed as a nondestructive quantum probe where a photon is detected, stored, and regenerated without touching its—-potentially undetermined—polarization.
\end{abstract}

PACS numbers: 42.50.Ex, 32.80.Qk, 42.50.Dv, 42.50.Nn

Among systems for the storage of light, one can distinguish between a device that can retain an initially prepared, and thus known, state [1,2], a quantum receiver that can receive and retain any (unknown) incoming state [3], and a quantum memory that can receive, retain, and recreate any (unknown) incoming state for further processing [4-7]. The attribute "quantum" indicates that the device outperforms any "classical" device (that attempts to measure and recreate the state) when averaged randomly over the Hilbert space of operation [3]. For instance, a classical polarization memory for single photons has a fidelity limit of $\frac{2}{3}$ since a single measurement allows only incomplete characterization of an unknown input state.

Quantum communication can benefit from quantum receivers and memories [8-10]. Moreover, the detrimental effects of photon loss can be largely remedied by a heralding ("state purification" [8]) feature that announces photon arrival and storage without destroying or revealing the stored quantum state. Heralded quantum memories may thus advance long-distance quantum communication [810], linear-optics quantum computing [11], and schemes aimed at breaking quantum encryption [12], or convert probabilistic $[2,13,14]$ into heralded entanglement.

A continuous-variable quantum receiver has been realized by Julsgaard et al. [3] who mapped a weak coherent electromagnetic field containing up to eight photons onto the collective spin of an atomic ensemble with a fidelity of $\mathcal{F}=0.67$ after $0.7 \mathrm{~ms}$ of storage. Recently, squeezed vacuum has been stored in and retrieved from an atomic ensemble [15], and a weak coherent state has been stored in a single atom [5].

Work with quantized excitations demonstrated capture and release of a single photon of fixed polarization [16,17] and coherent adiabatic transfer of a single photon between two ensembles via an optical resonator [18]. Matsukevich and Kuzmich [1] first introduced two ensembles, each capable of storing a single photon of fixed polarization, as a two-state system that can be mapped onto a single photon of variable polarization [19]. The two states can also be implemented as momentum states of the stored spin wave [2] or, as in the present work, using atoms in two different magnetic sublevels. The latter allows the direct mapping of the spin polarization onto a single spatial mode of an optical resonator.

The first system capable of functioning as a quantum memory was realized by Choi et al., who mapped the polarization state of an incoming photon onto two ensembles, and later retrieved the photon [6]. This (unheralded) device was tested for a single input polarization, for which it achieved a fringe visibility of 0.91 at a photon retrieval probability of 0.17 and a lifetime of $8 \mu \mathrm{s}$. A recent teleportation experiment by Chen et al. can also be viewed as a quantum memory, where the polarization state of an incoming photon is erased in a two-photon measurement, and teleported with probability between $17 \%$ and $95 \%$ onto two atomic ensembles [7] at polarization fidelities $\mathcal{F}$ between 0.74 and 0.87 . If this experiment were performed with a single input photon, heralded storage would occur with a probability of $\sim 10^{-4}$.

In this Letter, we demonstrate a heralded quantum memory where a single photon announces polarization storage in the form of a single collective-spin excitation (magnon) that is shared between two spatially overlapped atomic ensembles. The heralded storage occurs rarely $\left(h \approx 10^{-6}\right.$ per photon in our nonoptimized setup), but when it does, the stored photon can later be recreated with good efficiency $(\varepsilon \approx 0.5)$ and sub-Poissonian statistics $\left(g_{2}=0.24\right)$, while its polarization state is restored with very high fidelity $(\mathcal{F}>0.9)$. In the absence of a second setup for the production of narrowband single photons $[13,17,20]$, we test the quantum memory with coherent states of arbitrary polarization (containing typically 500 photons per pulse to improve the data collection rate). The scheme, however, is designed for single-quantum storage and stores only one photon even for coherent input beams [8,21].

Heralded storage is achieved by means of a spontaneous Raman process that simultaneously creates the herald and the magnon. To store an arbitrary polarization state 


$$
|\psi\rangle=\cos \theta|R\rangle+e^{i \phi} \sin \theta|L\rangle,
$$

written as a superpositions of right (left) circularly polarized states $|R\rangle(|L\rangle)$ with two arbitrary angles $\theta, \phi$, we use two spatially overlapped atomic ensembles $A, B$ inside an optical resonator. The atomic levels are chosen such that ensemble $A(B)$ absorbs only $|R\rangle(|L\rangle)$ polarized light, while both can emit a photon of the same polarization $(\pi)$ into the resonator on the Raman transition of interest [Fig. 1]. The detection of the emitted $\pi$ photon heralds the mapping of the input polarization state onto a magnon, but does not provide "which-path" information to distinguish between $A$ and $B$. The "write" process thus maps a polarization state $|\psi\rangle$ onto a magnon superposition state

$$
|\psi\rangle \rightarrow|\Psi\rangle=\cos \theta|1\rangle_{A}|0\rangle_{B}+e^{i \phi} \sin \theta|0\rangle_{A}|1\rangle_{B},
$$

where $|n\rangle_{k}$ denotes $n$ magnons in ensemble $k(k=A, B)$. At a later time, the stored state can be retrieved on demand as a single photon by utilizing the strong coupling of the magnon to the resonator $[8,21]$ ("read" process).

The heralding serves to enhance the fidelity of the write process by announcing successful events. In our present nonoptimized setup, the heralding probability per incoming photon is $h=\alpha \eta q \approx 10^{-6}$, where $\alpha=0.01$ is the absorption probability, $\eta=10^{-3}$ is the single atom cooperativity (the emission probability into the resonator in this case), and $q=0.1$ is the photon detection efficiency. Whenever there is a heralding event, however, a single magnon corresponding to the input-field polarization is stored with high fidelity and can be retrieved with good efficiency. The single-photon nature of the retrieved field is confirmed by a conditional autocorrelation measurement indicating sub-Poissonian statistics $\left[g_{2}=0.24(5)<1\right]$. The heralding process may thus be viewed as a quantum nondemolition measurement where a single photon is detected and stored while preserving its polarization.

The quantum memory uses precessing spins [22] in order to take advantage of resonator emission in both heralding and read processes, and of atomic symmetries; the former provides mode selection and high photon collection efficiency and the latter good polarization fidelity. We choose a $\pi$ transition for heralding, while any input state is expressed as a superposition of $\sigma^{ \pm}$polarizations [Fig. 1(b)]. Given the corresponding atomic angular emission patterns, we then need to rotate the atomic-spin direction by $90^{\circ}$ between the heralding and the readout. This is achieved with a magnetic field of $\sim 1.4 \mathrm{G}$ that induces Larmor spin precession with a period of $\tau_{L}=$ $2 \mu \mathrm{s}$ [Fig. 1(a) and 1(c)], enabling us to access the same magnon with different light polarizations at different times. Note that a spatially homogeneous magnetic field maintains the interatomic coherence and does not affect the magnon momentum, or equivalently, the phase matching condition for the read process [23].

We load cesium atoms from a magneto-optical trap into a far-detuned (trap wavelength $\lambda_{t}=1064 \mathrm{~nm}$ ) one-dimensional optical lattice overlapped with

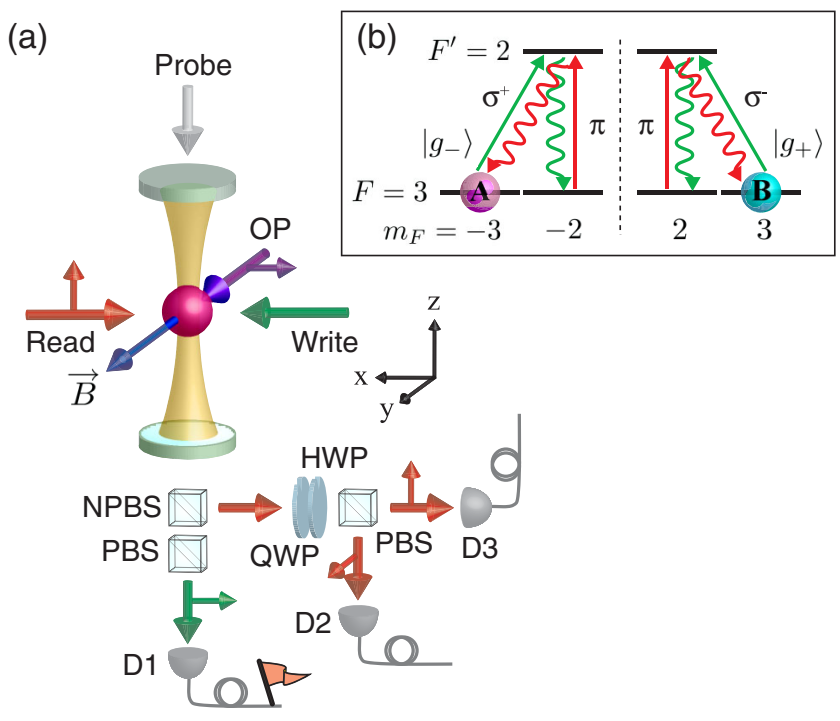

(c)

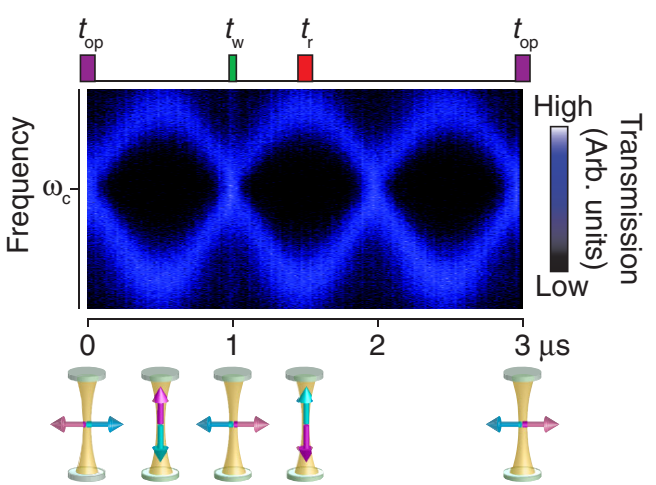

FIG. 1 (color online). (a) Setup. The small arrows indicate beam polarizations. OP is the optical pumping beam. NPBS, PBS, QWP, and HWP denote a nonpolarizing beam splitter, a polarizing beam splitter, a quarter wave plate, and a half wave plate, respectively. D1, D2, D3 are single-photon counting modules for herald detection and polarization analysis. A static magnetic field induces magnon precession. (b) Energy levels. Ensembles $A$ and $B$ are initially prepared in $\left|g_{\mp}\right\rangle \equiv$ $\left|F=3, m_{F}=\mp 3\right\rangle$. The write (green) and the read (red) processes are $\sigma^{ \pm}-\pi$ and $\pi-\sigma^{ \pm}$spontaneous Raman transitions, respectively. (c) Precession of the two macroscopic spins, as measured via cavity transmission, and timing of the opticalpumping $\left(t_{\mathrm{op}}\right)$, write $\left(t_{w}\right)$, and read $\left(t_{r}\right)$ processes.

the mode of a medium-finesse $(f=140)$ optical resonator [21]. Ensembles $A$ and $B$ consist of approximately 8000 atoms each at a temperature of $30 \mu \mathrm{K}$, optically pumped into hyperfine and magnetic sublevels $\left|g_{ \pm}\right\rangle \equiv$ $\left|6 S_{1 / 2}, F=3, m_{F}= \pm 3\right\rangle$, respectively, in the rotating frame. (The quantization axis is defined to rotate with the atomic spins and coincide with the propagation direction of the write beam at the optical-pumping time $t_{\mathrm{op}}=0$.) Optical pumping is achieved by periodic application of a short $\left(100 \mathrm{~ns} \ll \tau_{L}\right)$, linearly $\left(\hat{\mathbf{x}}_{-}-\right)$polarized opticalpumping pulse, resonant with the $6 S_{1 / 2}, F=3 \rightarrow 6 P_{3 / 2}$, $F^{\prime}=2$ transition. The ensembles $A, B$ thus form macroscopic spins in opposite directions that Larmor precess in 
the $x-z$ plane with a period $\tau_{L}$ [Fig. 1(c)]. We choose a pumping period of $\tau_{\mathrm{op}}=3 \tau_{L} / 2=3 \mu \mathrm{s}$, such that the ensembles are interchanged at every trial, which reduces population imbalance between $\left|g_{ \pm}\right\rangle$.

The atomic-spin precession and the efficiency of the optical pumping are monitored via resonator transmission of a weak, linearly $(\hat{\mathbf{x}}-)$ polarized beam. In the frame rotating with the atomic spin, the probe beam polarization, and thus the coupling to the polarized atoms, change periodically with time. Since the states $\left|g_{ \pm}\right\rangle$do not couple to $\pi$-polarized light on the chosen transition $F=3 \rightarrow$ $F^{\prime}=2$ [see Fig. 1(c)], the otherwise observable atominduced splitting of the cavity resonance [24] disappears. By optimizing the sinusoidal variation of the splitting [Fig. 1(c)], we prepare more than $99 \%$ of the $F=3$ population in either of the $\left|g_{ \pm}\right\rangle$sublevels.

The photon storage and readout processes are timed to match the sample precession [Fig. 1(c)]. A sequence of optical-pump, write, and read pulses is applied once every $\tau_{\text {op }}$ for $30 \mathrm{~ms}$, corresponding to a total of $10^{4}$ trials before the sample is recooled.

The light whose polarization state is to be stored (write beam) is tuned to the $F=3 \rightarrow F^{\prime}=2$ atomic transition, propagates along the $\hat{\mathbf{x}}$ direction, and is pulsed on for $50 \mathrm{~ns} \ll \tau_{L}$ at $t_{w}=\tau_{L} / 2=1 \mu \mathrm{s}$, when the macroscopic spins are aligned along $\mp \hat{\mathbf{x}}$. At this time, ensembles $A$ and $B$ can absorb only $|R\rangle\left(\sigma^{+}\right)$and $|L\rangle\left(\sigma^{-}\right)$photons, respectively [Fig. 1(b)]. For equal populations in $A$ and $B$, a $\pi$-polarized photon originating from a spontaneous $\sigma^{ \pm}-\pi$ (absorbing a $\sigma^{ \pm}$photon and emitting a $\pi$ photon) Raman process has the same probability for having been emitted by either ensemble. Thus, it does not provide any "which-path" information, and, if detected by detector D1 [Fig. 1(a)], serves as a herald that announces the storage of a (not revealed) polarization state $|\psi\rangle$ as a magnon $|\Psi\rangle$.

At $t_{r}=t_{w}+\tau_{L} / 4=1.5 \mu \mathrm{s}$, when the atomic spins point along the resonator axis $\pm \hat{\mathbf{z}}$, the $\hat{\mathbf{z}}$-polarized read beam, tuned to the $F=3 \rightarrow F^{\prime}=2$ transition, is applied for $100 \mathrm{~ns} \ll \tau_{L}$. The read beam addresses a $\pi$ transition, such that collectively enhanced [8] $\pi-\sigma^{ \pm}$Raman scattering maps the magnon state onto a single-photon polarization state. If the populations, $|\cos \theta|^{2},|\sin \theta|^{2}$, and the relative phase $\phi$ of the magnons in ensembles $A, B$ are preserved between the write and read processes [Eq. (2)], the polarization of the regenerated single photon is a faithful copy of the write beam polarization.

To quantify the performance of the heralded memory, we determine the density matrix $\rho_{\text {meas }}$ of the output polarization [examples are shown in Fig. 2(a)] by measurements in three polarization bases [25]: $\frac{1}{\sqrt{2}}(|L\rangle \pm|R\rangle)(H-V),|L\rangle$ and $|R\rangle(L-R)$, and $\frac{1}{\sqrt{2}}(|L\rangle \pm i|R\rangle)(S-T)$. The polarization fidelities $\mathcal{F}$ of the retrieved single photons for ten states of varying angle $\theta$ [Fig. 3] as well as for the six fiducial input states, $H, V, L, R, S$, and $T$ [Fig. 2], are evaluated as $\mathcal{F}=$ $\operatorname{Tr}\left(\rho_{\text {meas }}|\psi\rangle\langle\psi|\right)$, where $|\psi\rangle$ is the input state in Eq. (1). Figure 3 shows that $\mathcal{F}$ is close to unity with no systematic

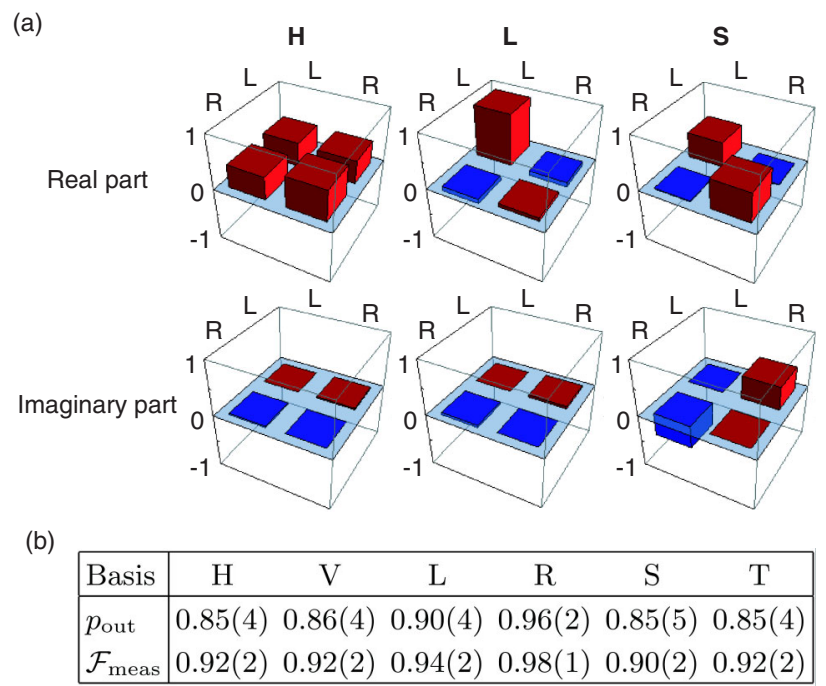

FIG. 2 (color online). (a) Density matrices $\rho_{\text {meas }}$ of the retrieved single photons for fiducial input states $H, L$, and $S$. (b) The measured degrees of polarization $\left(p_{\text {out }}\right)$ and fidelities $(\mathcal{F})$ of the retrieved single photons.

dependence on the zenith angle $\theta$, and we have verified separately that the same is true for the azimuth angle $\phi$. For any of the six fiducial states, the measured fidelity $\mathcal{F}$ without any background subtraction is significantly above the classical limit of $2 / 3$ for state-independent storage [Fig. 2(b)]. [The larger fluctuation of the fidelities in Fig. 3 (measured over $\sim 8$ hours) relative to Fig. 2(b) (measured over $\sim 1$ hour) is due to a slow uncompensated magnetic field drift.] If we correct the fidelities presented in Fig. 2(b) for the effect of the independently measured photon backgrounds, all fidelities are unity within statistical errors of a few percent.

The major source of photon backgrounds is the finite Larmor precession of $0.3 \mathrm{rad}$ during the 100 -ns read process. The read pump beam acquires a small admixture of $\sigma^{ \pm}$components in the frame precessing with the atomic

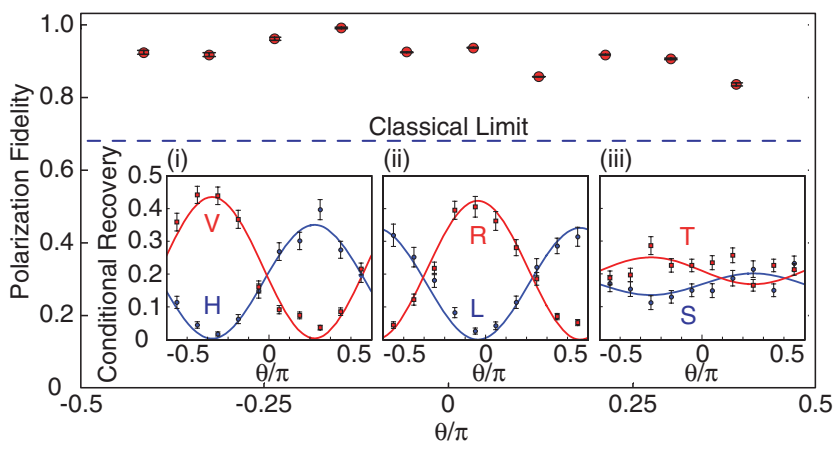

FIG. 3 (color online). Polarization fidelity of the stored photon as a function of $\theta$ for $\phi=0$ [Eq. (1)]. Insets (i)-(iii): The results of projection measurements of the output field in three mutually orthogonal bases, $H-V, L-R$, and $S-T$. The solid curves are a simultaneous fit for all 60 data points. No backgrounds have been subtracted. 
spin [Fig. 1(b)], which results in strong resonator emission by atoms in $\left|g_{ \pm}\right\rangle$. These backgrounds deteriorate not only the stored polarization, but also the single-photon character of the retrieved field. The predicted autocorrelation due to this effect is $g_{2} \approx 0.2$, in good agreement with the measured value of $g_{2}=0.24(5)$. These backgrounds can be reduced by slowing down the Larmor precession, which requires an increase in the memory lifetime which is currently $\sim 3 \mu \mathrm{s}$. With a lifetime of a few milliseconds as demonstrated recently [26], the autocorrelation will then be $g_{2} \sim 0.02$, limited only by the rare storage of two photons. The current lifetime is set by the magnon Doppler decoherence [21] that is presently faster than polarization (spin) decoherence. The one-dimensional optical lattice in the setup does not confine the atoms along the direction of momentum transfer in the write process [21], and thus an additional lattice is required for long lifetime.

Finally, we discuss the performance limits for the heralded quantum memory scheme investigated here. The success probability may be improved upon by adding a second resonator to increase the absorption probability of the incident photon. The fundamental limit for the heralding probability $h$ for $N \gg 1$ would then be given by $h=$ $\frac{\eta}{1+\eta} q$. Under realistic conditions $(N=100, \eta=10, q=$ 0.6 ), one could thus achieve $h \sim 0.5$ for an incident single photon with retrieval efficiencies near 90\% [21]. With a lifetime of $\sim 6 \mathrm{~ms}$ [26], Larmor precession and spin decoherence would limit the polarization fidelity only at the $10^{-3}$ level.

By applying this scheme to photons of undetermined polarization from a probabilistic source of entangled photon-magnon pairs $[2,13,14]$, it should be possible to realize a heralded source of entangled-photon pairs for various tasks in quantum information processing. If loaded by a photon from a high-purity Bell pair, the estimated Bell parameter for the heralded source employing our current quantum memory would be $2.5(1)>2$, exceeding the classical limit. For the above improved parameters, such a source would then produce heralded Bell pairs at a rate of $\sim 500 \mathrm{~s}^{-1}$.

We gratefully acknowledge support by the NSF and DARPA. J. S. thanks the NDSEG and NSF for support.

[1] D. Matsukevich and A. Kuzmich, Science 306, 663 (2004).

[2] S. Chen, Y.-A. Chen, B. Zhao, Z.-S. Yuan, J. Schmiedmayer, and J.-W. Pan, Phys. Rev. Lett. 99, 180505 (2007).

[3] B. Julsgaard, J. Sherson, J. I. Cirac, J. Fiurášek, and E. S. Polzik, Nature (London) 432, 482 (2004).

[4] A. A. Kiselev, K. W. Kim, and E. Yablonovitch, Appl. Phys. Lett. 80, 2857 (2002).
[5] A. D. Boozer, A. Boca, R. Miller, T. E. Northup, and H. J. Kimble, Phys. Rev. Lett. 98, 193601 (2007).

[6] K. Choi, H. Deng, J. Laurat, and H. Kimble, Nature (London) 452, 67 (2008).

[7] Y.-A. Chen, S. Chen, Z.-S. Yuan, B. Zhao, C.-S. Chuuand, J. Schmiedmayer, and J.-W. Pan, Nature Phys. 4, 103 (2008).

[8] L.-M. Duan, M. D. Lukin, J. I. Cirac, and P. Zoller, Nature (London) 414, 413 (2001).

[9] L. Jiang, J. M. Taylor, and M. D. Lukin, Phys. Rev. A 76, 012301 (2007).

[10] Z.-B. Chen, B. Zhao, Y.-A. Chen, J. Schmiedmayer, and J.-W. Pan, Phys. Rev. A 76, 022329 (2007).

[11] E. Knill, R. Laflamme, and G. Milburn, Nature (London) 409, 46 (2001).

[12] G. Brassard, N. Lütkenhaus, T. Mor, and B.C. Sanders, Phys. Rev. Lett. 85, 1330 (2000).

[13] D. N. Matsukevich, T. Chanelière, M. Bhattacharya, S.-Y. Lan, S.D. Jenkins, T.A.B. Kennedy, and A. Kuzmich, Phys. Rev. Lett. 95, 040405 (2005).

[14] H. de Riedmatten, J. Laurat, C. W. Chou, E. W. Schomburg, D. Felinto, and H. J. Kimble, Phys. Rev. Lett. 97, 113603 (2006).

[15] K. Honda, D. Akamatsu, M. Arikawa, Y. Yokoi, K. Akiba, S. Nagatsuka, T. Tanimura, A. Furusawa, and M. Kozuma, Phys. Rev. Lett. 100, 093601 (2008); J. Appel, E. Figueroa, D. Korystov, M. Lobino, and A. I. Lvovsky, Phys. Rev. Lett. 100, 093602 (2008).

[16] T. Chaneliere, D. N. Matsukevich, S. D. Jenkins, S. Y. Lan, T. A. B. Kennedy, and A. Kuzmich, Nature (London) 438, 833 (2005).

[17] M.D. Eisaman, A. André, F. Massou, M. Fleischhauer, A. S. Zibrov, and M. D. Lukin, Nature (London) 438, 837 (2005).

[18] J. Simon, H. Tanji, S. Ghosh, and V. Vuletić, Nature Phys. 3, 765 (2007).

[19] C. W. Chou, H. de Riedmatten, D. Felinto, S. V. Polyakov, S. J. van Enk, and H. J. Kimble, Nature (London) 438, 828 (2005).

[20] J. K. Thompson, J. Simon, H. Loh, and V. Vuletić, Science 313, 74 (2006).

[21] J. Simon, H. Tanji, J. K. Thompson, and V. Vuletić, Phys. Rev. Lett. 98, 183601 (2007).

[22] D. N. Matsukevich, T. Chanelière, S. D. Jenkins, S.-Y. Lan, T. A. B. Kennedy, and A. Kuzmich, Phys. Rev. Lett. 96, 033601 (2006).

[23] A. T. Black, J. K. Thompson, and V. Vuletić, Phys. Rev. Lett. 95, 133601 (2005).

[24] Y. Zhu, D. J. Gauthier, S.E. Morin, Q. Wu, H. J. Carmichael, and T.W. Mossberg, Phys. Rev. Lett. 64, 2499 (1990).

[25] D. F. V. James, P. G. Kwiat, W. J. Munro, and A. G. White, Phys. Rev. A 64, 052312 (2001).

[26] B. Zhao, Y.-A. Chen, X.-H. Bao, T. Strassel, C.-S. Chuu, X.-M. Jin, J. Schmiedmayer, Z.-S. Yuan, S. Chen, and J.-W. Pan, Nature Phys. 5, 95 (2009); R. Zhao, Y. O. Dudin, S. D. Jenkins, C. J. Campbell, D. N. Matsukevich, T. A. B. Kennedy, and A. Kuzmich, Nature Phys. 5, 100 (2009). 